\title{
Correction to: Long-term shifts in the growth and maturation size of Miyabe charr Salvelinus malma miyabei
}

\author{
Yuki Yamamoto $^{1,2} \cdot$ Taku Yoshiyama $^{1} \cdot$ Keitaro Kajiwara ${ }^{1} \cdot$ Toshikuni Nakatani $^{1} \cdot$ Takashi Matsuishi $^{3}$ (I)
}

Published online: 25 April 2018

C) Japanese Society of Fisheries Science 2018

\section{Correction to: Fisheries Science https://doi.org/10.1007/s12562-018-1186-5}

In the original publication the first author's name is incorrect. The author's correct name is provided in this article. The original article has been corrected.

The original article can be found online at https://doi.org/10.1007/ s12562-018-1186-5.

Takashi Matsuishi

catm@fish.hokudai.ac.jp

Yuki Yamamoto

yukiyamamoto2705@gmail.com

Taku Yoshiyama

yoshiyama@fish.hokudai.ac.jp

Keitaro Kajiwara

kajiwarakeitaro@gmail.com

Toshikuni Nakatani

nakatani@fish.hokudai.ac.jp

1 Faculty of Fisheries Sciences, Hokkaido University,

Hakodate, Hokkaido, Japan

2 Present Address: Graduate School of Agricultural Science,

Tohoku University, 468-1 Aramaki Aza Aoba, Aoba-ku,

Sendai, Miyagi 980-0845, Japan

3 Global Institution for Collaborative Research and Education,

Faculty of Fisheries Sciences, Hokkaido University, 3-1-1

Minato-cho, Hakodate, Hokkaido 041-8611, Japan 